\title{
Der Datenschutz und die Weitergabe medizinischer Daten an die Versicherer
}

T. Mattiga, C. Lutz $^{b}$
Damit ein Versicherer einen Versicherungsantrag prüfen oder seine Leistungspflicht in einem konkreten Fall abklären kann, ist er auf Informationen und die Mitwirkung des behandelnden Arztes* angewiesen. Die Weitergabe von medizinischen Daten an die Versicherer ist jedoch für die Ärzte heikel, handelt es sich hierbei doch um besonders schützenswerte Daten im Sinne des Datenschutzgesetzes (DSG). Zu Recht fragen sich die Ärzte, ob sie derartige Informationen überhaupt weitergeben dürfen, und was mit diesen anschliessend passieren wird.

\section{Rechtmässige Datenweitergabe}

Alle Informationen über den Patienten, welche der Arzt in Ausübung seiner beruflichen Tätigkeit erfährt, fallen unter das Arztgeheimnis, d.h., grundsätzlich darf der Arzt solche Daten weder mündlich noch schriftlich an Dritte weitergeben. Liegt hingegen ein Rechtfertigungsgrund vor, sei es, dass die betroffene Person eingewilligt hat, oder dass die Informationsbeschaffung durch Gesetz gerechtfertigt ist, so ist die Datenweitergabe rechtmässig. Die Unfallversicherer nach Unfallversicherungsgesetz (UVG) haben eine klare gesetzliche Grundlage für die Informationsbeschaffung. Dasselbe gilt für die Krankenversicherer nach Krankenversicherungsgesetz (KVG). Die übrigen Versicherer müssen sich auf eine Einwilligung der versicherten oder geschädigten Person stützen.

Am Beispiel der Krankenversicherung ergibt dies folgende zwei Varianten:

1. Der Arzt als Leistungserbringer nach KVG ist im Verhältnis zum Krankenversicherer von seinem Berufsgeheimnis befreit (Art. 42 Abs. 3 und 4 und Art. 57 Abs. 6 KVG) und untersteht einer gesetzlichen Auskunftspflicht. Dies gilt für die Grundversicherung.

2. Im Bereich der Zusatzversicherungen muss der Arzt durch die versicherte Person von der Schweigepflicht entbunden werden.

Korrespondenz:

Dr. iur. Thomas Mattig

Schweizerischer

Versicherungsverband SVV

Conrad F. Meyer-Strasse 14

CH-8022 Zürich

Tel. 012082828
Somit darf und muss der Arzt Informationen an den UVG-Versicherer, an den KVG-Versicherer und - falls eine entsprechende Einwilligung gegeben wurde - auch an die übrigen Versicherer

\footnotetext{
E-Mail: thomas.mattig@svv.ch weiterleiten.
}

\section{Verhältnismässigkeit der Datenbearbeitung}

Gemäss Datenschutzgesetz muss jede Datenbearbeitung verhältnismässig sein. Es dürfen nur jene Daten bearbeitet werden, die für die Erfüllung des bestimmten Zweckes erforderlich sind, wobei die Persönlichkeitsbeeinträchtigung möglichst geringzuhalten ist. Liegt eine gültige Einwilligung des Patienten vor, so ergibt sich aus deren Wortlaut der Umfang der Daten, die rechtmässig verlangt werden können.

Die privaten UVG-Versicherer und die Suva müssen sich nicht auf eine Einwilligung berufen. Sie können gestützt auf das UVG alle für die Feststellung der Leistungspflicht erforderlichen Daten einholen. In der Unfallversicherung herrscht die Untersuchungsmaxime, d.h. der Sachverhalt ist von Amtes wegen sehr genau $\mathrm{zu}$ ermitteln. So hat der Unfallversicherer beispielsweise festzustellen, ob überhaupt ein Unfallereignis stattgefunden hat, ob Vorzustände zu berücksichtigen sind usw.

Für die Krankenversicherer gilt die Untersuchungsmaxime ebenfalls. Sie dürfen sich auf der Grundlage des KVG all jene Daten beschaffen, die zur Erfüllung ihrer Aufgabe erforderlich sind. In diesem Zusammenhang ist immer wieder daran zu erinnern, dass die Krankenversicherer keine blossen Zahlstellen sind, sondern sie müssen zuerst ihre Leistungspflicht und ihren Leistungsumfang überprüfen und in einem zweiten Schritt die Wirksamkeit, Zweckmässigkeit und Wirtschaftlichkeit der Leistungen.

\section{Behandlung von irrelevanten oder hochsensiblen Daten}

Von Datenschutzseite wird befürchtet, die Versicherer würden sich - zur Verfolgung anderer Zwecke - in grossen Mengen Daten beschaffen, die sie für ihre Aufgabenerfüllung gar nicht benötigen. Dies verstosse gegen das Verhältnismässigkeitsprinzip, wonach bei der Bestellung von medizinischen Daten immer fein säuberlich zwischen relevanten und irrelevanten Angaben getrennt werden müsse.

Tatsächlich interessieren sich die Versicherer jedoch nur für jene Informationen, die sie ent- 
weder für die Antragsprüfung oder für die Schadenerledigung brauchen. Auch ihnen geht es letztlich darum, jeden überflüssigen administrativen Aufwand zu vermeiden. Im übrigen stellt sich die Frage, wie ein Versicherer die Relevanz einer Information beurteilen soll, wenn er diese gar nicht kennt. Jedenfalls müsste er seine Abklärungen oft mittels umfangreicher Fragebögen treffen, was zu einer Aufblähung des Verwaltungsapparates führen würde. Auch für die Ärzteschaft wäre ein solcher Aufwand kaum zu bewältigen. Überzogene Forderungen an die Verhältnismässigkeit sind nicht praxistauglich und sie führen auch nicht zu einer spürbaren Verbesserung des Datenschutzes. Vielmehr kann man einerseits darauf vertrauen, dass der Arzt keine offensichtlich irrelevanten Daten weiterleitet. Andererseits ist der Versicherer gehalten, ihm zugeschickte Daten zu vernichten, wenn diese für die Aufgabenerfüllung nicht erforderlich sind.

Bei den Krankenversicherern nach KVG sorgt der Vertrauensarzt für eine zusätzliche Dimension des Datenschutzes: Er erfüllt eine eigentliche Filterfunktion für «Daten mit einem erhöhten Schutzbedürfnis». Demgegenüber haben die Privatversicherer keine Vertrauensärzte, sondern sie arbeiten mit beratenden Ärzten zusammen. Auch hier können aber hochsensible Daten ausnahmsweise an den «medizinischen Dienst» des Versicherers adressiert werden. In aller Regel ist jedoch eine Zustellung der Informationen an den Sachbearbeitenden unbedenklich.

\section{Bearbeitung der Patientendaten durch die Versicherer}

Für die Handhabung medizinischer Akten durch die Versicherer ergeben sich aus dem Datenschutzgesetz ebenfalls strenge Auflagen. Wiederum ist das Verhältnismässigkeitsprinzip entscheidend: Datenzugriff soll nur haben, wer die entsprechenden Informationen auch tatsächlich braucht. Alle Versicherer sehen intern vor, dass Papier- und elektronische Daten nur einem beschränkten Personenkreis zur Verfügung stehen.
Für Daten in Papierform wird dies vor allem erreicht, indem die Fälle einzelnen Sachbearbeitenden zugeteilt werden und das Archiv nicht allgemein zugänglich ist. Die elektronischen Zugriffe werden für die verschiedenen Datensammlungen unterschiedlich - gestützt auf die jeweiligen Bedürfnisse - geregelt. Alle Versicherer sind zudem dafür verantwortlich, dass keine besonders schützenswerten Personendaten an unberechtigte Dritte gelangen. Sie schulen die Mitarbeitenden entsprechend und treffen die notwendigen organisatorischen und technischen Vorkehrungen.

\section{Fazit}

Die Weitergabe von medizinischen Daten an die Versicherer ist immer dann rechtmässig, wenn eine Einwilligung der betroffenen Person oder eine Rechtfertigung durch Gesetz vorliegt, wie dies im UVG und im KVG der Fall ist. Die Versicherer dürfen lediglich jene Daten bearbeiten, die sie für die Erfüllung ihrer Aufgabe benötigen. Offensichtlich irrelevante Informationen muss der Arzt daher nicht weiterleiten. Ausserdem kann er besonders heikle Daten dem Vertrauensarzt der KVG-Versicherer und in Ausnahmefällen auch dem «medizinischen Dienst» der Privatversicherer zustellen. Die Datenbearbeitung durch die Versicherer unterliegt ebenfalls strengen datenschutzrechtlichen Kriterien.

\section{Literatur}

- Hürlimann B, Jacobs R, Poledna T (Hrsg.). Datenschutz im Gesundheitswesen. Zürich: Schulthess; 2001.

- Brühwiler-Frésey LS. Medizinischer Behandlungsvertrag und Datenrecht. Zürich: Schulthess; 1996.

- Keller K. Das ärztliche Berufsgeheimnis gemäss Art. 321 StGB. Dissertation. Zürich, Schulthess; 1993.

- Günter P. Datenschutz im Gesundheitsbereich. In: Schweizer RJ (Hrsg.). Das neue Datenschutzgesetz des Bundes. Zürich: Referate der Tagungen der HSG vom 15. Oktober und 13. November 1992; 1993. 